"Supporting Information"

\title{
Carbon-supported platinum electrocatalysts probed in a gas diffusion setup with alkaline environment: how particle size and mesoscopic environment influence the degradation mechanism
}

Shima Alinejad ${ }^{\mathrm{a}}$, Jonathan Quinson ${ }^{\mathrm{b}}$, Johanna Schröder ${ }^{\mathrm{a}}$, Jacob J. K. Kirkensgaard ${ }^{\mathrm{c}}$, Matthias Arenz ${ }^{\mathrm{a}^{*}}$

${ }^{a}$ Department of Chemistry and Biochemistry, University of Bern, Freiestrasse 3, 3012 Bern, Switzerland

${ }^{\mathrm{b}}$ Department of Chemistry, University of Copenhagen, Universitetsparken 5, 2100 Copenhagen $\emptyset$, Denmark

${ }^{c}$ Department of Food Science, University of Copenhagen, Rolighedsvej 26, 1958 Frederiksberg, Denmark

*Corresponding author

University of Bern, Freiestrasse 3, CH-3012 Bern, Switzerland

Phone: +41316315384

Email: matthias.arenz@dcb.unibe.ch

In table S1, the initial electrochemical surface area (ECSA) of the $4-5 \mathrm{~nm} \mathrm{Pt} / \mathrm{C}$ catalyst is shown under different measurement conditions. In the first two rows, the ECSA of the 4-5 nm Pt/C in a conventional liquid cell, rotating disk electrode (RDE), and gas diffusion electrode (GDE) is compared. Thereafter, the comparison of the ECSA in the GDE setup by changing the following parameters can be discussed: the ionomer in the ink formulation from the Nafion dispersion (D1021, $10 \mathrm{wt} \%$, EW 1100, Fuel Cell Store) to an anion ionomer (Sustainion® XA-9 Alkaline Ionomer 5\% in ethanol, Dioxide Materials), the membrane from a Nafion exchange membrane (Nafion 117, $183 \mu \mathrm{m}$ thick, Fuel Cell Store) to different types of anion exchange membrane, and 
the electrolyte from $70 \%$ perchloric acid $\left(\mathrm{HClO}_{4}\right.$, Suprapur, Merck) to the potassium hydroxide (KOH, Merck).

For the anion exchange membrane, many different types from different commercial stores were tried; such as Sustainion grade T (Sustainion ${ }^{\circledR}$ X37-50 Grade T Membrane, Dioxide Materials), Sustainion grade RT (Sustainion ${ }^{\circledR}$ X37-50 Grade RT Membrane, with a dry thickness of $50 \mu \mathrm{m}$ thick, Dioxide Materials), Zirfon Perl (Zirfon Perl UTP 500 Membrane, with a thickness of 500 $\mu \mathrm{m}$, Agfa), and Fumasep (Fumasep FAB-PK-130, with a thickness of $130 \mu \mathrm{m}$, fuelcellstore).

Table S1. The initial surface area of the 4-5 $\mathrm{nm} \mathrm{Pt/C}$ catalyst is reported under different measurement conditions.

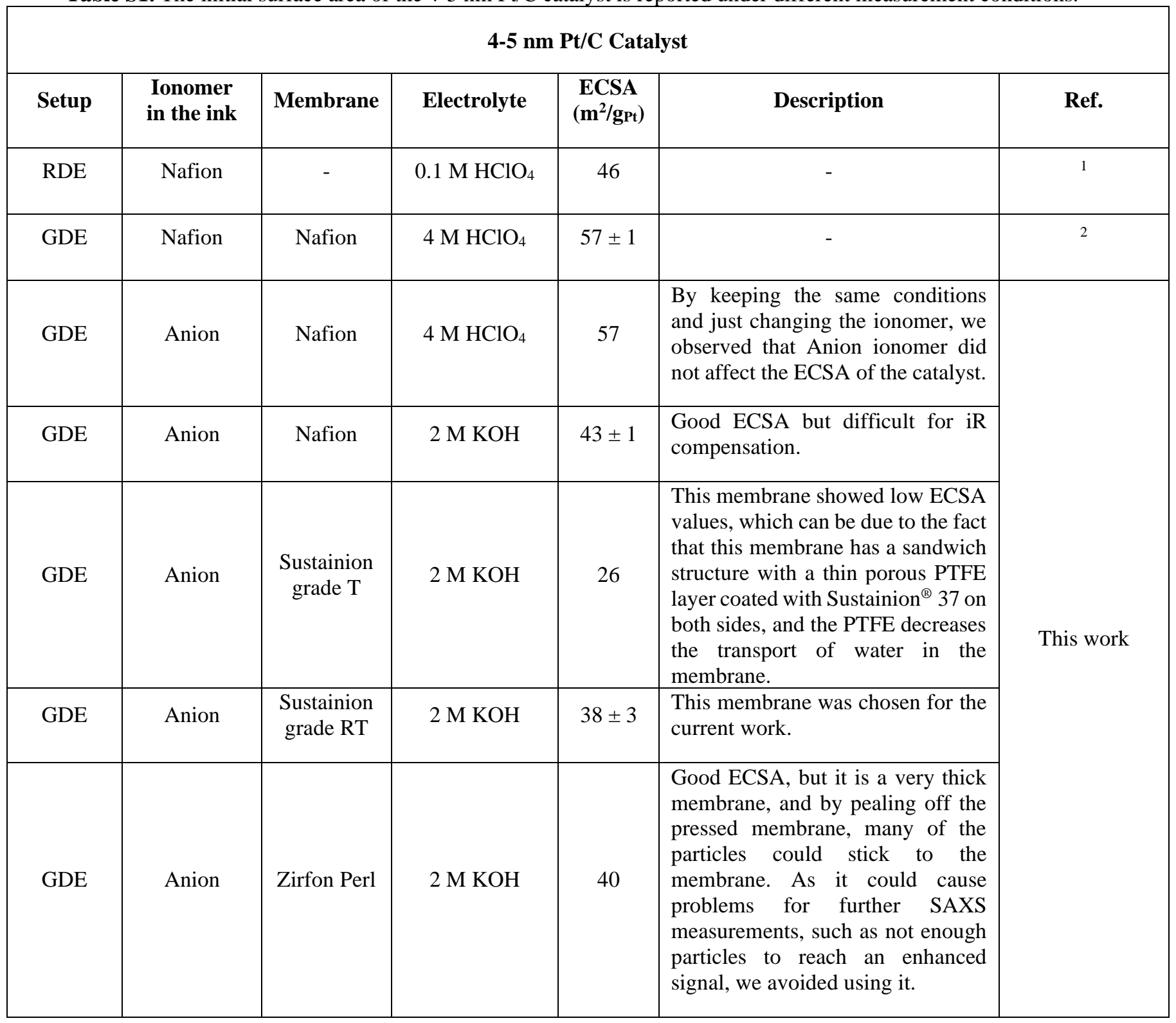




\begin{tabular}{|c|c|c|l|l|l|}
\hline GDE & Anion & $\begin{array}{c}\text { Fumasep } \\
\text { FAB-PK- } \\
130\end{array}$ & $2 \mathrm{M} \mathrm{KOH}$ & - & $\begin{array}{l}\text { This membrane showed very high } \\
\text { resistance (above 1000 ohm), and } \\
\text { because of this reason, it was not } \\
\text { possible to perform the } \\
\text { measurements. }\end{array}$ \\
\hline
\end{tabular}

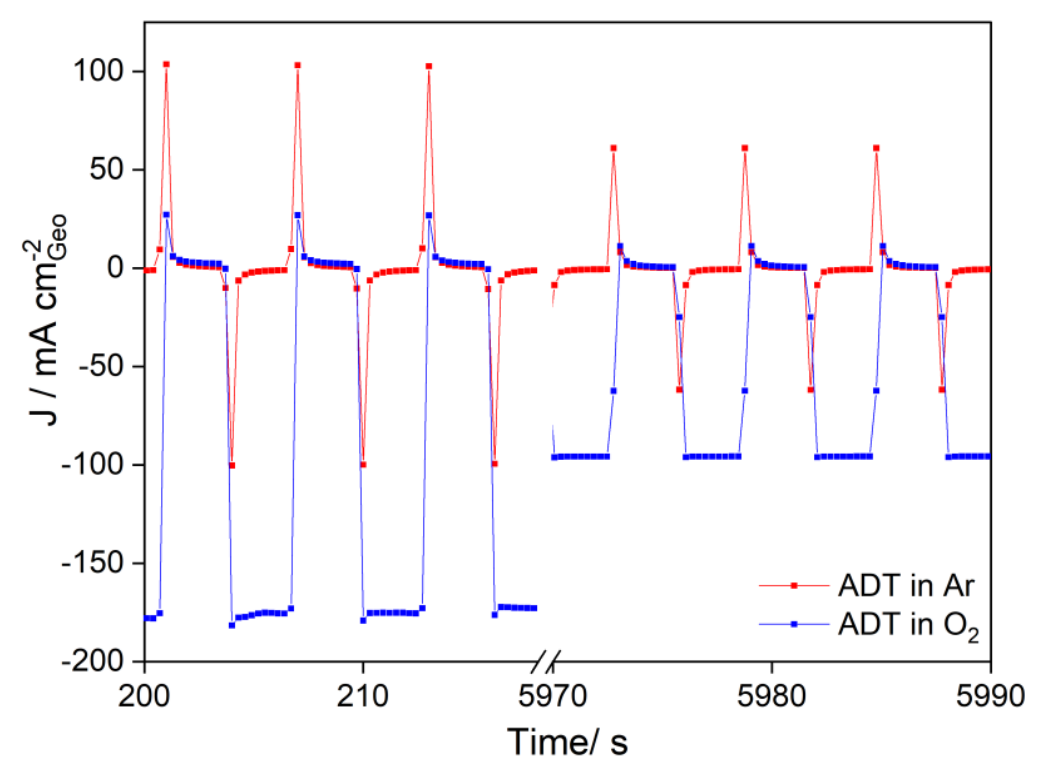

Figure S1. Representative current densities recorded during applying the ADT protocol in Ar atmosphere (red) and $\mathrm{O}_{2}$ atmosphere (blue) at the beginning (left) and at the end (right) of the protocol. It is seen that in $\mathrm{O}_{2}$ atmosphere substantially higher currents flow due to the ORR. 


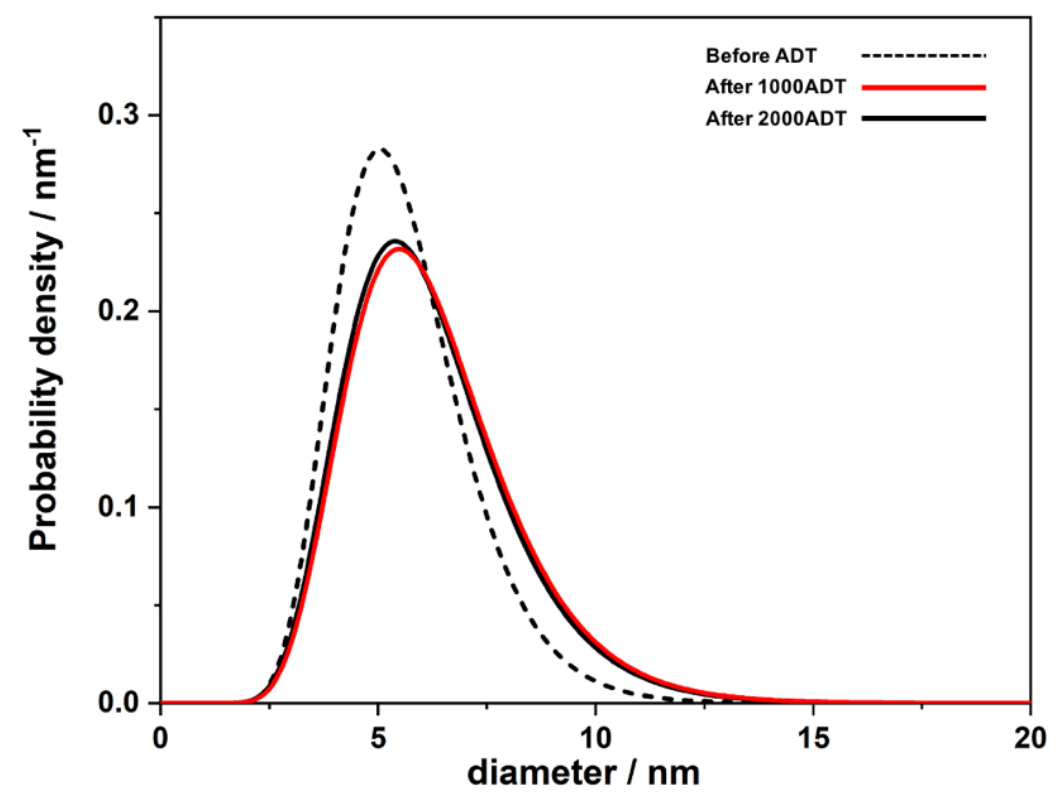

Figure S2. Representative SAXS particle size distributions of 4-5 nm Pt/C catalyst before (dashed lines), after 1000 and 2000 steps of ADTs in Ar (red and black lines, respectively).

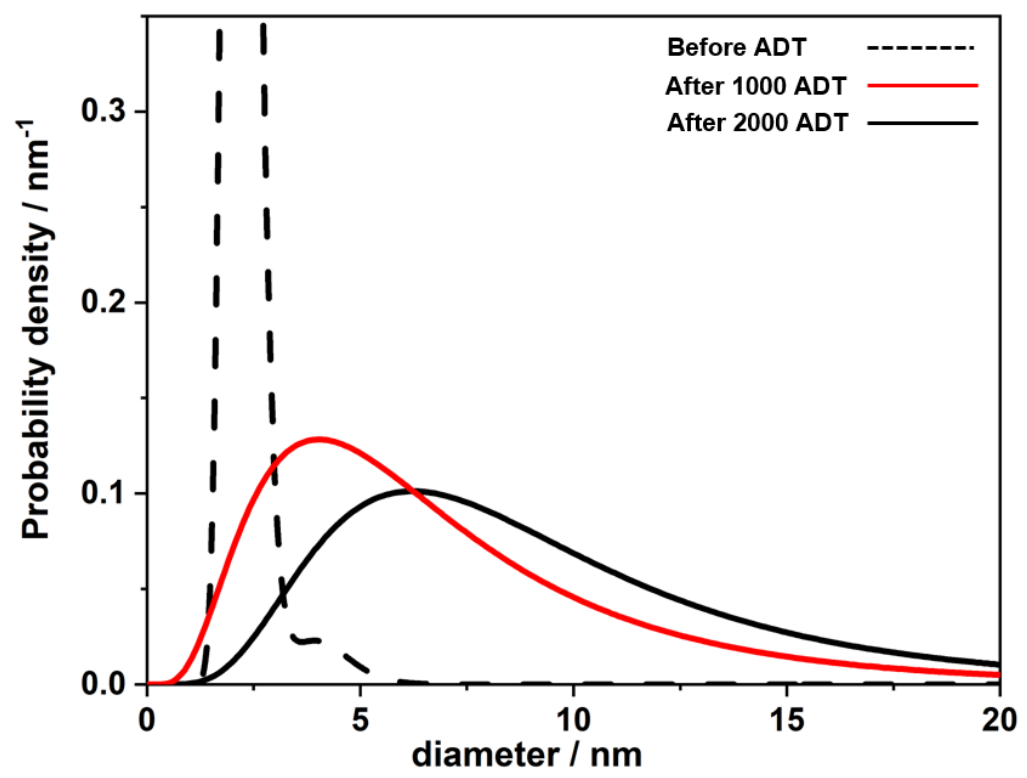

Figure S3. Representative SAXS particle size distributions of bimodal catalyst before (dashed lines), after 1000 and 2000 steps of ADTs in Ar atmosphere (red and black lines, respectively).

\section{SAXS data}

The average volume of nanoparticle from population 1 and $2,\langle\mathrm{~V}\rangle_{1}$ and $\langle\mathrm{V}\rangle_{2}$ respectively, define volume fraction of population 1 and $2, \Phi_{\mathrm{v} 1}$ and $\Phi_{\mathrm{v} 2}$, respectively as: 


$$
\begin{gathered}
\Phi_{\mathrm{V} 1}=\frac{\mathrm{N}_{1}<\mathrm{V}>_{1}}{\mathrm{~N}_{1}<\mathrm{V}>_{1}+\mathrm{N}_{2}<\mathrm{V}>_{2}}=1-\Phi_{\mathrm{V} 2} \\
\frac{\Phi_{\mathrm{V} 1}}{\Phi_{\mathrm{V} 2}}=\frac{\mathrm{N}_{1}<\mathrm{V}>_{1}}{\mathrm{~N}_{2}<\mathrm{V}>_{2}} \\
\frac{\mathrm{N}_{1}}{\mathrm{~N}_{2}}=\frac{\Phi_{\mathrm{V} 1}<\mathrm{V}>_{2}}{\Phi_{\mathrm{V} 2}<\mathrm{V}>_{1}}
\end{gathered}
$$

where $\mathrm{N}_{1}$ and $\mathrm{N}_{2}$ are the number of nanoparticles in the population 1 and 2 , respectively.

The relationship between the retrieved coefficient $\mathrm{C}_{1}$ and $\mathrm{C}_{2}$ is given by: $\mathrm{C}_{\mathrm{i}}=\mathrm{k} . \Phi_{\mathrm{vi}} .\langle\mathrm{V}\rangle_{\mathrm{i}}$ where $\mathrm{i}=1$ or 2 and $\mathrm{k}$ is a constant.

$$
\begin{gathered}
\mathrm{k}=\frac{\mathrm{C}_{1}}{\Phi_{\mathrm{V} 1}<\mathrm{V}>_{1}}=\frac{\mathrm{C}_{2}}{\Phi_{\mathrm{V} 2}<\mathrm{V}>_{2}}=\frac{\mathrm{C}_{2}}{\left(1-\Phi_{\mathrm{V} 1}\right)<\mathrm{V}>_{2}} \\
\frac{\Phi_{\mathrm{V} 1}}{1-\Phi_{\mathrm{V} 1}}=\frac{\mathrm{C}_{1}<\mathrm{V}>_{2}}{\mathrm{C}_{2}<\mathrm{V}>_{1}} \\
\Phi_{\mathrm{V} 1}=\frac{1}{1+\frac{\mathrm{C}_{2}<\mathrm{V}>_{1}}{\mathrm{C}_{1}<\mathrm{V}>_{2}}}
\end{gathered}
$$

In order to weight the probability density function by the area or surface fractions we consider $\langle\mathrm{A}\rangle_{1}$ and $\langle\mathrm{A}\rangle_{2}$ as the average area of the nanoparticles from population 1 and 2 , respectively:

$$
\begin{gathered}
\Phi_{\mathrm{A} 1}=\frac{\mathrm{N}_{1}<\mathrm{A}>_{1}}{\mathrm{~N}_{1}<\mathrm{A}>_{1}+\mathrm{N}_{2}<\mathrm{A}>_{2}}=1-\Phi_{\mathrm{A} 2}=\frac{1}{1+\frac{\mathrm{N}_{2}<\mathrm{A}>_{2}}{\mathrm{~N}_{1}<\mathrm{A}>_{1}}} \\
\Phi_{\mathrm{A} 1}=\frac{1}{1+\frac{\Phi_{\mathrm{V} 2}<\mathrm{V}>_{1}<\mathrm{A}>_{2}}{\Phi_{\mathrm{V} 1}<\mathrm{V}>_{2}<\mathrm{A}>_{1}}} \\
\Phi_{\mathrm{A} 1}=\frac{1}{1+\frac{C_{2}\left(<\mathrm{V}>_{1}\right)^{2}<\mathrm{A}>_{2}}{C_{1}\left(<\mathrm{V}>_{2}\right)^{2}<\mathrm{A}>_{1}}}
\end{gathered}
$$


Table S2. SAXS data fits and size analysis.

\begin{tabular}{|c|c|c|c|c|c|c|c|c|c|c|c|c|c|c|}
\hline \multirow{2}{*}{\multicolumn{2}{|c|}{ Samples }} & \multirow{2}{*}{\multicolumn{2}{|c|}{ Power law }} & \multirow{2}{*}{\multicolumn{3}{|c|}{$1^{\text {st }}$ population }} & \multirow{2}{*}{\multicolumn{3}{|c|}{$2^{\text {nd }}$ population }} & \multirow{2}{*}{\multicolumn{5}{|c|}{ Size and distribution }} \\
\hline & & & & & & & & & & & & & & \\
\hline & & Ax $10^{6}$ & $\mathrm{n}$ & $\begin{array}{l}\mathrm{R}_{1} \\
(\AA)\end{array}$ & $\sigma_{1}$ & $\mathrm{C}_{1}$ & $\begin{array}{l}\mathrm{R}_{2} \\
(\AA)\end{array}$ & $\sigma_{2}$ & $\mathrm{C}_{2}$ & $\begin{array}{c}\mathrm{d}_{\text {sample }}{ }^{\mathrm{A}} \\
{[\mathrm{nm}]}\end{array}$ & $\sigma_{\text {sample }}{ }^{\mathrm{B}}[\mathrm{nm}]$ & $\begin{array}{l}\text { diameter: } \\
<\mathrm{d}\rangle^{\mathrm{C}}[\mathrm{nm}]\end{array}$ & $\begin{array}{c}\text { of }<\mathrm{d}>\text { : } \\
\sigma_{\mathrm{d}-\mathrm{i}, \mathrm{ii}, \mathrm{iii} i \mathrm{iv}} \\
{[\mathrm{nm}]}\end{array}$ & $\begin{array}{c}\text { on }<\mathrm{d}>\text { : } \\
\sigma_{<\mathrm{d}>}{ }^{E}[\mathrm{~nm}]\end{array}$ \\
\hline \multirow{12}{*}{$1-2 \mathrm{~nm}$} & \multirow{4}{*}{$\begin{array}{c}\text { As } \\
\text { prepared }\end{array}$} & 700 & 3.00 & 9.8 & 0.12 & 0.0045 & & & & 2.0 & 0.2 & \multirow{4}{*}{2.0} & \multirow{4}{*}{0.1} & \multirow{4}{*}{0.01} \\
\hline & & 210 & 3.00 & 9.8 & 0.12 & 0.0034 & & & & 2.0 & 0.2 & & & \\
\hline & & 630 & 3.00 & 9.8 & 0.11 & 0.0042 & & & & 2.0 & 0.2 & & & \\
\hline & & 470 & 3.05 & 9.6 & 0.18 & 0.0044 & & & & 2.0 & 0.4 & & & \\
\hline & \multirow{4}{*}{$\begin{array}{c}\text { Ar } \\
2000 \text { cycles }\end{array}$} & 90 & 3.5 & & & & 33.0 & 0.55 & 0.020 & 7.7 & 4.6 & \multirow{4}{*}{9.0} & \multirow{4}{*}{3.7} & \multirow{4}{*}{1.36} \\
\hline & & 108 & 3.3 & & & & 37.0 & 0.75 & 0.034 & 9.8 & 8.5 & & & \\
\hline & & 170 & 3.0 & & & & 38.0 & 0.80 & 0.035 & 10.5 & 9.9 & & & \\
\hline & & 10 & 3.8 & & & & 34.3 & 0.55 & 0.018 & 8.0 & 4.7 & & & \\
\hline & \multirow{4}{*}{$\begin{array}{c}\mathrm{O}_{2} \\
1000 \text { cycles }\end{array}$} & 55 & 3.4 & & & & 18.0 & 0.40 & 0.002 & 3.9 & 1.6 & \multirow{4}{*}{5.6} & \multirow{4}{*}{1.9} & \multirow{4}{*}{2.18} \\
\hline & & 120 & 3.3 & & & & 18.5 & 0.45 & 0.007 & 4.1 & 1.9 & & & \\
\hline & & 6 & 4.0 & & & & 36.0 & 0.60 & 0.027 & 8.6 & 5.7 & & & \\
\hline & & 17 & 4.0 & & & & 22.0 & 0.70 & 0.019 & 5.6 & 4.5 & & & \\
\hline \multirow{10}{*}{ 4-5 nm } & \multirow{3}{*}{$\begin{array}{c}\text { As } \\
\text { prepared }\end{array}$} & 1.8 & 4.0 & & & & 27.1 & 0.27 & 0.022 & 5.6 & 1.5 & \multirow{3}{*}{5.6} & & \\
\hline & & 5.1 & 4.0 & & & & 27.1 & 0.28 & 0.021 & 5.6 & 1.6 & & 0.9 & 0.01 \\
\hline & & 3 & 4.0 & & & & 27.0 & 0.28 & 0.023 & 5.6 & 1.6 & & & \\
\hline & $\mathrm{Ar}$ & 4 & 3.8 & & & & 31.3 & 0.26 & 0.009 & 6.5 & 1.7 & & & \\
\hline & 2000 cycles & 140 & 3.4 & & & & 29.5 & 0.30 & 0.018 & 6.2 & 1.9 & 6.3 & 1.3 & 0.21 \\
\hline & $\mathrm{Ar}$ & 6.5 & 4.0 & & & & 30.0 & 0.30 & 0.019 & 6.3 & 1.9 & & & \\
\hline & 1000 cycles & 11 & 3.8 & & & & 30.0 & 0.24 & 0.013 & 6.2 & 1.5 & 6.3 & 1.2 & 0.07 \\
\hline & & 210 & 3.2 & & & & 28.0 & 0.45 & 0.007 & 6.2 & 2.9 & & & \\
\hline & $\begin{array}{c}\mathrm{O}_{2} \\
1000 \mathrm{cycles}\end{array}$ & 140 & 3.3 & & & & 29.0 & 0.32 & 0.016 & 6.1 & 2.9 & 6.4 & 1.9 & 0.46 \\
\hline & & 18 & 3.3 & & & & 29.0 & 0.60 & 0.012 & 6.9 & 4.6 & & & \\
\hline & & 52 & 3.3 & 9.8 & 0.17 & 0.0022 & 27.5 & 0.35 & 0.016 & $2.0 / 5.8$ & $0.3 / 2.1$ & & & \\
\hline & & 30 & 3.3 & 8.8 & 0.20 & 0.0029 & 26.0 & 0.34 & 0.015 & $1.8 / 5.5$ & $0.4 / 1.9$ & 1.9 & 0.2 & 0.12 \\
\hline & prepared & 200 & 3.3 & 8.6 & 0.20 & 0.0037 & 27.0 & 0.34 & 0.020 & $1.8 / 5.7$ & $0.4 / 2.0$ & 57 & 10 & 014 \\
\hline & & 160 & 3.3 & 9.6 & 0.20 & 0.0040 & 27.0 & 0.32 & 0.020 & $2.0 / 5.7$ & $0.4 / 1.9$ & 5.7 & 1.0 & 0.14 \\
\hline & & 50 & 3.6 & & & & 32.0 & 0.35 & 0.016 & 6.8 & 2.5 & & & \\
\hline Mixed & Ar & 150 & 3.4 & & & & 31.0 & 0.30 & 0.023 & 6.5 & 2.0 & 6.7 & 1.2 & 0.19 \\
\hline & & 390 & 3.2 & & & & 33.0 & 0.25 & 0.023 & 6.8 & 1.7 & & & \\
\hline & & 48 & 3.6 & & & & 28.0 & 0.55 & 0.018 & 6.5 & 3.9 & & & \\
\hline & & 8 & 4.0 & & & & 30.0 & 0.45 & 0.018 & 6.6 & 3.1 & & & \\
\hline & 1000 cycles & 130 & 3.2 & & & & 30.0 & 0.40 & 0.011 & 6.5 & 2.7 & 6.8 & 2.0 & 0.43 \\
\hline & & 3.5 & 4.0 & & & & 30.0 & 0.65 & 0.007 & 7.4 & 5.4 & & & \\
\hline & & 120 & 3.0 & 10.5 & 0.13 & 0.0021 & 18.5 & 0.20 & 0.0012 & $2.1 / 3.8$ & $0.3 / 0.8$ & 2.2 & 0.2 & 0.08 \\
\hline & As & 80 & 3.0 & 11.0 & 0.15 & 0.0029 & 20.5 & 0.17 & 0.001 & $2.2 / 4.2$ & $0.3 / 0.7$ & & & \\
\hline Bimodal & & 65 & 3.3 & 11.2 & 0.18 & 0.0033 & 22.5 & 0.20 & 0.001 & $2.3 / 4.6$ & $0.4 / 0.9$ & 4.2 & 0.4 & 0.41 \\
\hline catalyst & $\mathrm{Ar}$ & 20 & 3.2 & & & & 40.0 & 0.50 & 0.012 & 9.1 & 4.8 & & & \\
\hline & 2000 cycles & 300 & 2.6 & & & & 44.5 & 0.60 & 0.023 & $\begin{array}{l}9.1 \\
10.7\end{array}$ & $\begin{array}{l}4.0 \\
7.0\end{array}$ & 9.9 & 4.3 & 1.12 \\
\hline & $\mathrm{Ar}$ & 2 & 4.0 & & & & 35.0 & 0.60 & 0.005 & 8.4 & 5.5 & 7.3 & 3.5 & 1.55 \\
\hline
\end{tabular}




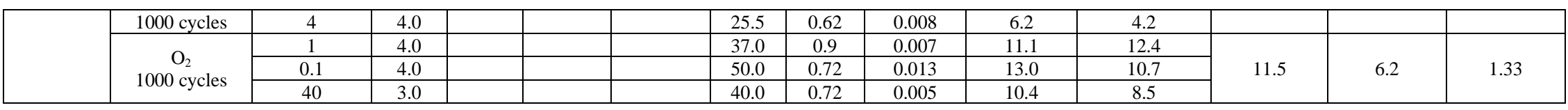

For the sample in grey a hard-sphere structure factor $F(R, \eta)$, see ${ }^{3}$ was used with $\eta=0.05$

(A) evaluated as $\mathrm{d}_{\text {sample }}=0.2 e^{\left(\ln (R)+\frac{\sigma^{2}}{2}\right)}$ for a one size population, evaluated as $\mathrm{d}_{\text {sample }}=0.2 \varphi_{\mathrm{A} 1} \cdot e^{\left(\ln \left(R_{1}\right)+\frac{\sigma_{1}{ }^{2}}{2}\right)}+0.2 \varphi_{\mathrm{A} 2} \cdot e^{\left(\ln \left(R_{2}\right)+\frac{\sigma_{2}}{2}\right)}$ for a 2 sizes population with $\mathrm{R}_{1}$ and $\mathrm{R}_{2}$ evaluated in $\AA$ and $\mathrm{d}_{\text {sample }}$ in $\mathrm{nm}$

(B) evaluated as $\sigma_{\text {sample }}=0.2 \sqrt{\left(e^{\sigma^{2}}-1\right) e^{\left(2 \ln (R)+\sigma^{2}\right)}}$ for a one size population,

evaluated as $\sigma_{\text {sample }}=0.2 \sqrt{\varphi_{\mathrm{A} 1}{ }^{2} \cdot\left[\left(e^{\sigma_{1}{ }^{2}}-1\right) e^{\left(2 \ln \left(R_{1}\right)+\sigma_{1}{ }^{2}\right)}\right]+\varphi_{\mathrm{A} 2}{ }^{2} \cdot\left[\left(e^{\sigma_{2}{ }^{2}}-1\right) e^{\left(2 \ln \left(R_{2}\right)+\sigma_{2}{ }^{2}\right)}\right]}$ for a two size population

(C) evaluated as the mean value of several measurements, for instance 4 measurements i, ii, iii and iv: $\langle\mathrm{d}\rangle=\frac{d_{i}+d_{i i}+d_{i i i}+d_{i v}}{4}$

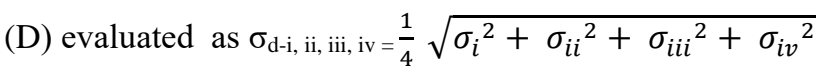

(E) evaluated as the deviation relative to the mean value diameter estimation from several measurements, this is a measure of how reproducible different measurements for a same condition are 

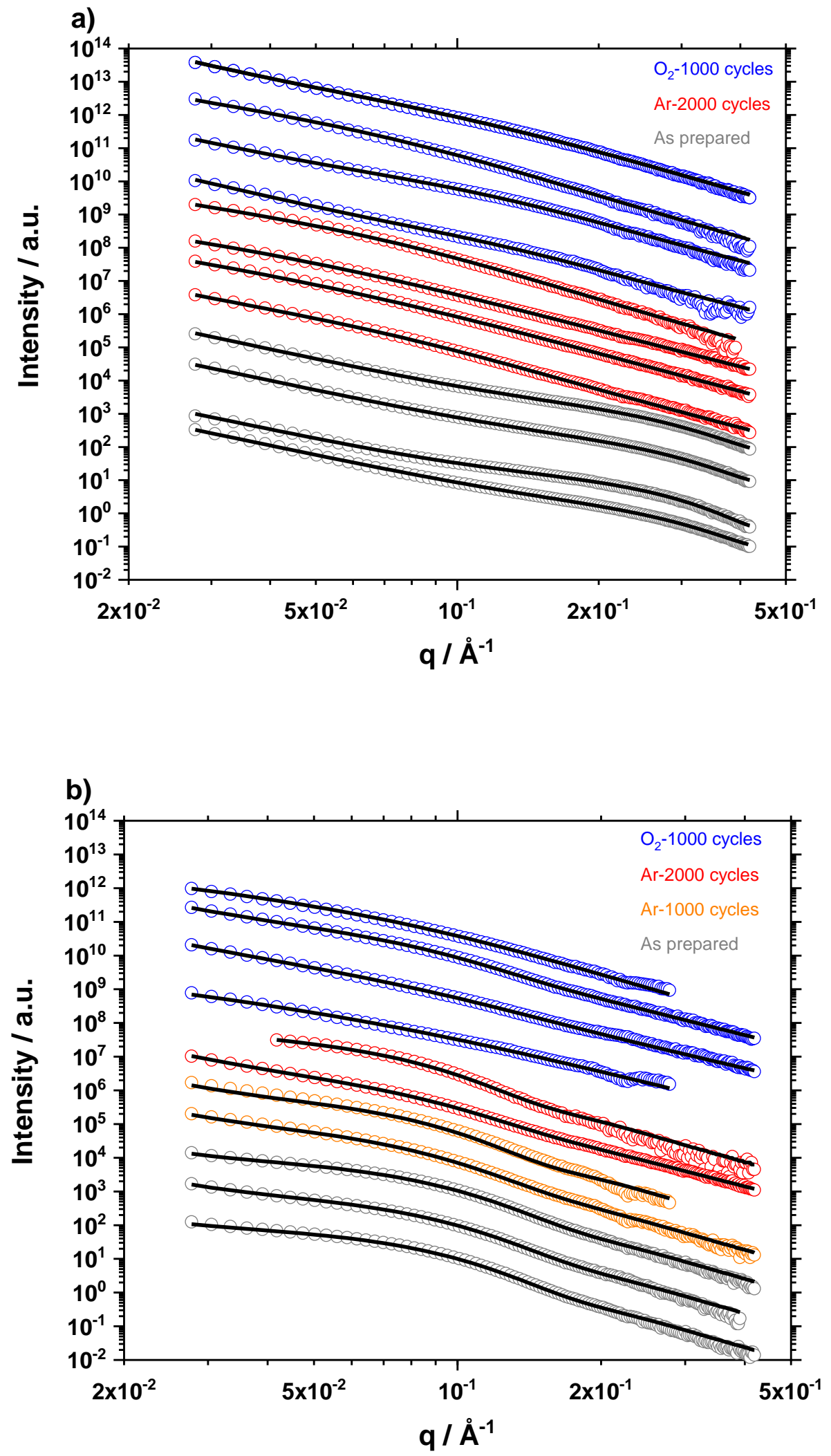

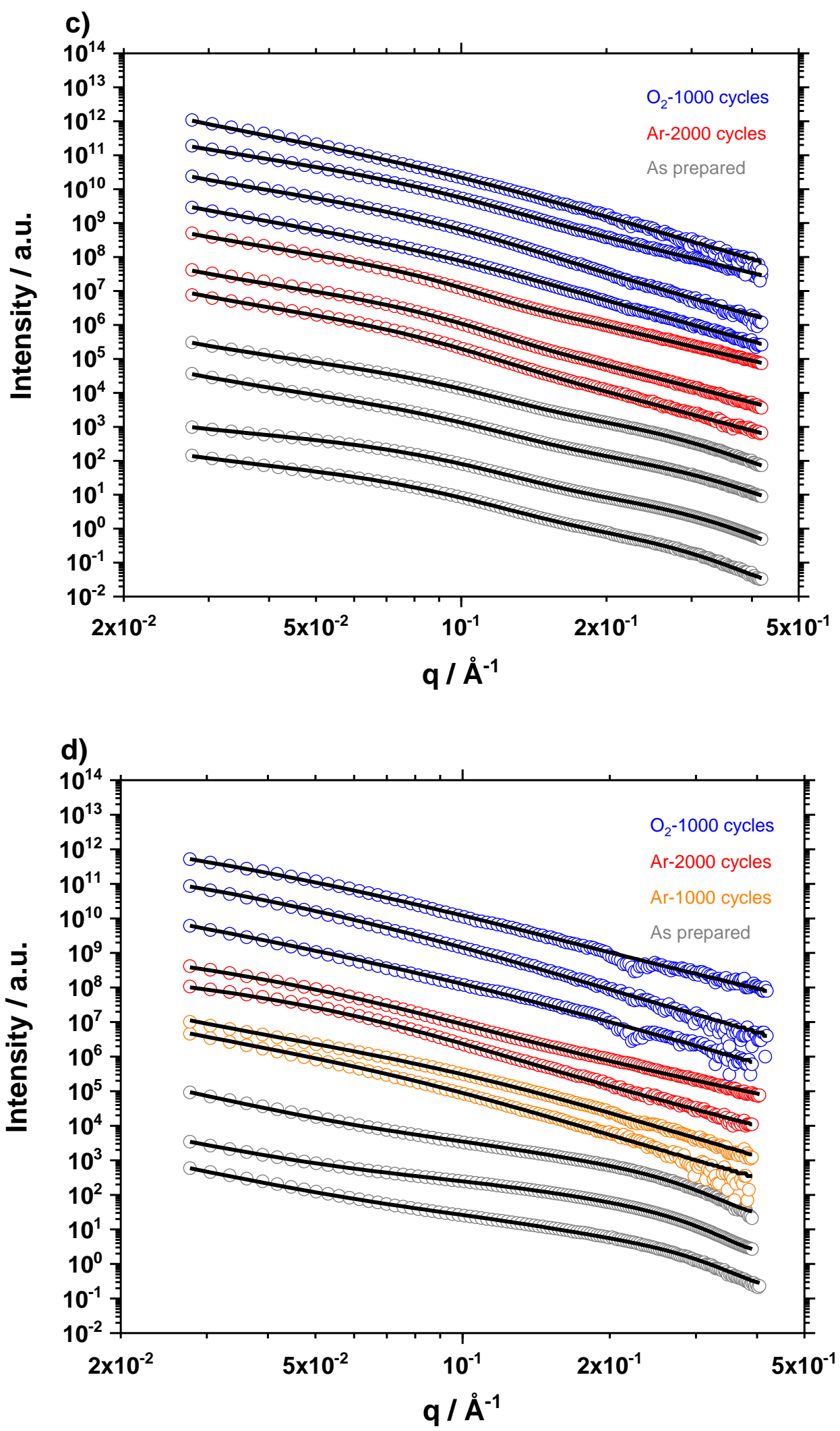

Figure S4. SAXS data and fits for the different conditions used as indicated and for (a) 1-2 nm (a), 4-5 $\mathrm{nm}(\mathrm{b})$, mixed (c), and bimodal (d) Pt/C catalysts. 


\section{References}

(1) Nesselberger, M.; Ashton, S.; Meier, J. C.; Katsounaros, I.; Mayrhofer, K. J. J.; Arenz, M. The Particle Size Effect on the Oxygen Reduction Reaction Activity of Pt Catalysts: Influence of Electrolyte and Relation to Single Crystal Models. J. Am. Chem. Soc. 2011, 133 (43), 17428-17433. https://doi.org/10.1021/ja207016u.

(2) Schröder, J.; Quinson, J.; Kirkensgaard, J. J. K.; Alinejad, S.; Mints, V. A.; Jensen, K. M. Ø.; Arenz, M. A New Approach to Probe the Degradation of Fuel Catalysts Under Realistic Conditions: Combining Tests in a Gas Diffusion Electrode Setup with Small Angle X-Ray Scattering. 2020, 120. https://doi.org/doi.org/10.26434/chemrxiv.12263804.

(3) Zemb, T.; Lindner, P. Neutron, X-Rays and Light. Scattering Methods Applied to Soft Condensed Matter; 1st Edition, Elsevier, 2002. ISBN: 9780444511225. 